\title{
Facile Preparation of Dehydrodigallic Acid and Its Derivative for the Synthesis of Ellagitannins
}

\author{
Kazuma Shioe, Shingo Ishikura, Yoshikazu Horino, and Hitoshi Abe* \\ Graduate School of Science and Engineering, University of Toyama; Toyama 930-8555, Japan. \\ Received June 10, 2013; accepted September 17, 2013
}

\begin{abstract}
A facile method for the synthesis of dehydrodigallic acid, which is a fundamental structure of ellagitannins, was developed. A classical Ullmann condition was effective for the formation of the highly hindered biaryl ether structure, and we clarified that the suitable protection of the phenolic hydroxy groups was crucial in this reaction. In this way, the synthesis of dehydrodigallic acid and its derivative was successfully performed. The described method would provide a synthetic utility toward ellagitannins.
\end{abstract}

Key words ellagitannin; Ullmann reaction; biaryl ether

Ellagitannins are representative polyphenolic secondary metabolites of higher plants and possess a wide range of biological activities (e.g., antioxidant, antivirus, and antitumor activities). ${ }^{1)}$ Although this class of compounds is composed only of gallic acid (1), oxidized gallic acid, and sugar, a few hundred congeners have been discovered in nature as the monoor oligomeric form (Fig. 1). Based on their multifunctional property and structural diversity, these compounds have attracted a great deal of attention from many synthetic chemists. One of the most outstanding features of the ellagitannnins is furnishing an axially chiral biaryl unit which is formed by the $\mathrm{C}-\mathrm{C}$ oxidative phenolic coupling of gallic acid (1), hence they produce ellagic acid (2) upon hydrolysis. From this point of view, synthetic studies of the ellagitannnins have focused on the construction of the biaryl part with an axial chirality ${ }^{2-5)}$; however, gallic acid (1) also provides dehydrodigallic acid (3) as a skeletal motif of the ellagitannins via the biogenetic $\mathrm{C}-\mathrm{O}$ oxidative coupling reaction in plants. In fact, numerous ellagitannnins incorporate this compound as the dehydrodigalloyl (DHDG) group in their structure, such as coriariin B. ${ }^{7)}$ Therefore, a convenient method for preparing $\mathbf{3}$ has been required to synthesize a diverse set of ellagitannins.

The synthesis of $\mathbf{3}$ was already accomplished by the classical Ullmann reaction of phenol and an aryl halide that was derived from 1. ${ }^{8)}$ In the $\mathrm{C}-\mathrm{O}$ bond formation step, however, the yield was very low. In 1996, Feldman and co-workers overcame the difficulty of the construction of the highly functionalized biaryl ether structure of $\mathbf{3}$ in a completely different way. $\left.{ }^{9}\right)$ They investigated the reactivity of galloyl-derived orthoquinones, such as $\mathbf{4}$, and applied it to the hetero DielsAlder reaction (Chart 1). The four-step sequence involving the Diels-Alder dimerization/reductive rearrangement was moderately effective for the synthesis of $\mathbf{5} \mathbf{a}$ that was the benzylated derivative of $\mathbf{3}$, which was the key synthetic intermediate of coriariin A. ${ }^{10-12)}$ Although their strategy is very effective for the ellagitannin synthesis, it might be difficult that the two ester groups of 5a would be distinguished. ${ }^{13)}$ This concern must be solved in order to synthesize coriariin B and other ellagitannins. In this context, we thus reinvestigated the synthesis of $\mathbf{3}$ and its derivative using the Ullmann condensation ${ }^{14)}$ that seemed to be convergent, flexible, and efficient, as shown in Chart $1 .^{15-18)}$<smiles>O=C(O)c1cc(O)c(O)c(O)c1</smiles>

gallic acid (1)<smiles>O=c1oc(=O)c2cc(O)c(O)c3c4oc(=O)c(c1cc(O)c4O)c23</smiles>

ellagic acid (2)<smiles>O=C(O)c1cc(O)c(O)c(Oc2c(O)c(O)cc(O)c2O)c1</smiles>

dehydrodigallic acid (3)

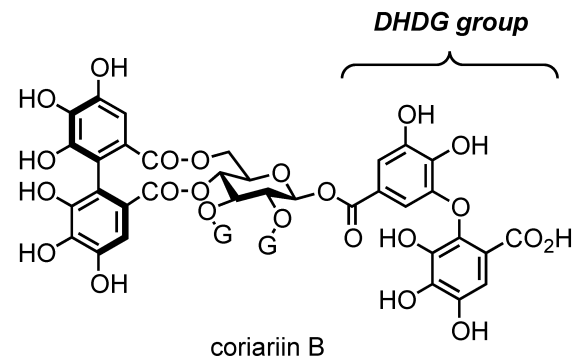<smiles>CC(=O)c1cc(O)c(O)c(CCCCO)c1</smiles>

Fig. 1. Structure of Ellagitannin-Related Compounds 
<smiles>COC(=O)C1=CC(=O)C(=O)C(Cc2ccccc2)=C1</smiles>

4

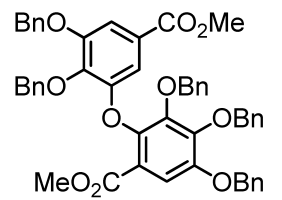

$5 a$<smiles>COC(=O)c1cc(OCc2ccccc2)c(OCc2ccccc2)c(OCc2ccccc2)c1Br</smiles>

$6 a$ 7

Chart 1. Two Different Approaches for Highly Functionalized Biaryl Ether<smiles>[R]Oc1cc(C(C)=O)cc(O)c1[R20]</smiles>

6a: $R=B n$

6b: $R=M e$<smiles>[R]C1Oc2cc(C(C)=O)cc(O)c2O1</smiles>

6c: $\mathrm{R}=\mathrm{OEt}$

6d: $R=P h$

6e: $R=P M P$
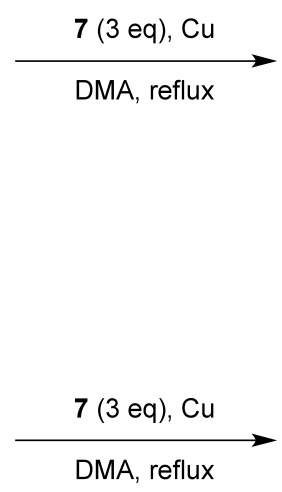

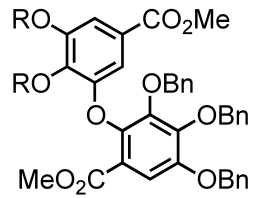

5a: $\mathrm{R}=\mathrm{Bn}(\sim 67 \%)$

5b: $R=\operatorname{Me}(79 \%)$<smiles></smiles>

5c: $R=$ OEt $(71 \%)$

5d: $\mathrm{R}=\mathrm{Ph} \quad(91 \%)$

5e: $R=\operatorname{PMP}(46 \%)$

Chart 2. Ullmann Reaction of $\mathbf{6 a}-\mathbf{e}$ with $\mathbf{7}$

For our initial explorations, the reaction of phenol $\mathbf{6} \mathbf{a}^{19)}$ and an aryl bromide $7^{20}$ was carried out as a model system. Although a small amount of 5a was detected in the presence of excess copper dust ${ }^{21)}$ and $N, N$-dimethylformamide (DMF), a serious amount of unavoidable byproducts, such as a dehalogenated compound of 7 and a homocoupling product of 7 were produced while the starting material $\mathbf{6 a}$ remained. This is a typical drawback of the classical Ullmann reaction. Thus, we examined another reagent system using copper(I) species. ${ }^{22)}$ However, the desired 5a was not obtained and debenzylation of the substrates was observed. This complexity might be due to the steric hindrance of the ortho-substituted 7. In this regard, we thought that there would be a possibility only using a less sterically-hindered compound as the coupling partner under the $\mathrm{Cu}(0)$-mediated classical Ullmann reaction to construct the sterically-hindered biaryl ether.

In order to consume phenol $\mathbf{6 a}$ and complete the reaction, we needed $3 \mathrm{eq}$ of the aryl bromide 7 with an excess amount of copper dust in a refluxing solvent. ${ }^{23)}$ When we used $\mathrm{N}, \mathrm{N}$ dimethylacetamide (DMA) as the solvent, 5a was successfully obtained in an acceptable yield (Chart 2). In this case, the dehalogenated byproduct produced from 7 was easily separated by standard silica gel column chromatography and 5a was isolated in an almost pure form. In order to improve the yield, we attempted to modify the protective group on the catechol

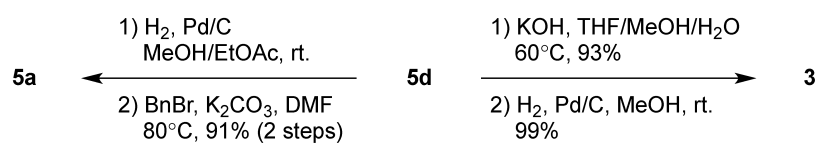

Chart 3. Synthesis of Dehydrodigallic Acid (3)

part of $\mathbf{6}$. When $\mathbf{6 b}^{24)}$ was subjected to the reaction, $\mathbf{5} \mathbf{b}$ was obtained in a slightly better yield. It was suggested that the steric hindrance of the phenol side was related to the reactivity. $^{2)}$ We postulated that an acetal type protective group, which can be easily attached to the phenolic hydroxyl groups, would be effective to decrease the steric hindrance. From this standpoint, phenols $\mathbf{6 c}-\mathbf{e}$ were prepared ${ }^{25-27)}$ and underwent the same reaction. It was found that benzylidene acetal $\mathbf{6 d}$ seemed to be the most suitable for this reaction than the orthoester 6c and $p$-methoxybenzylidene acetal $\mathbf{6 e}$. We thought that the lower yields of both $\mathbf{5 c}$ and $\mathbf{5 e}$ were due to the unstability of the acetal moiety under the reaction conditions. Consequently, the biaryl ether $\mathbf{5 d}$, which is an equivalent of $\mathbf{5} \mathbf{a}$, was obtained in a satisfactory yield (91\%), and thus, the reaction was carried out on a gram-scale basis.

With the biaryl ether $\mathbf{5 d}$ in hand, we attempted the synthesis of $\mathbf{3}$ (Chart 3). The transformation of $\mathbf{5 d}$ to Feldman's intermediate 5a was performed by a simple operation including hydrogenolysis and benzylation. Additionally, the synthesis 
<smiles>O=C(O)c1cc(O)c(O)c(C(=O)OCc2ccccc2)c1</smiles>

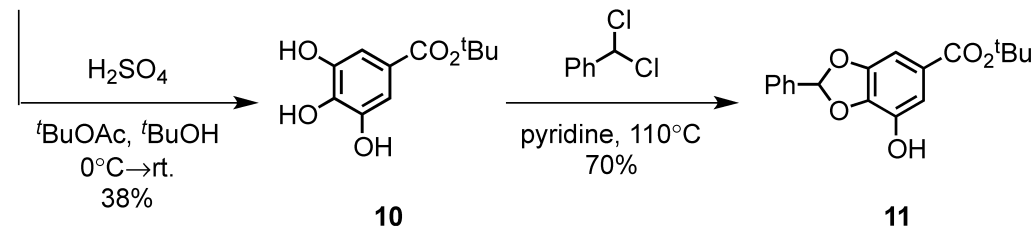

Chart 4. Functionalization of Gallic Acid (1)<smiles>CCCCOC(=O)c1cc(Oc2c(C(=O)OCc3ccccc3)cc(OC(C)(C)C)c(Br)c2Br)c2c(c1)OC(c1ccccc1)O2</smiles>

12

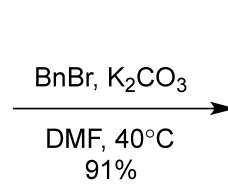

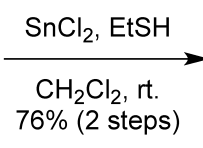

\%\% (2 steps)<smiles>CCOC(=O)c1cc(O)c(O)c(Oc2c(C(=O)OCc3ccccc3)cc(OCc3ccccc3)c(OCc3ccccc3)c2OCC(C)(C)C)c1</smiles>

13<smiles>CCC(C)OC(=O)c1cc(OCc2ccccc2)c(OCc2ccccc2)c(Oc2c(C(=O)OCc3ccccc3)cc(OCc3ccccc3)c(OC(C)(C)C)c2OCc2ccccc2)c1COCc1ccccc1</smiles>

14

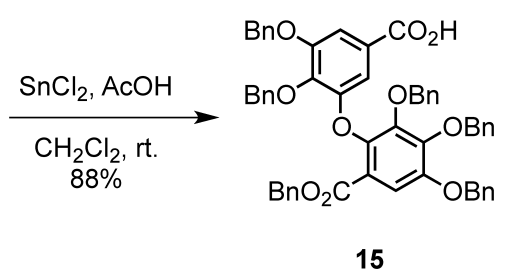

Chart 5. Synthesis of $\mathbf{1 5}$

of $\mathbf{3}$ was also accomplished from $\mathbf{5} \mathbf{d}$ by a two-step sequence that involved the hydrolysis of the methyl ester and reductive cleavage of the benzyl and benzylidene groups. The spectral data of the synthetic 3 was matched the reported data. ${ }^{28)}$

When we plan to synthesize the natural ellagitannin, coriariin B, the two carboxy groups of $\mathbf{3}$ should be distinguished for the construction of the dehydrodigalloyl part. In order to mask the carboxylic acid, we selected the benzyl and tertbutyl esters, which can be cleaved in a different deprotective manner. Chart 4 shows the preparation of the bromide 9 and phenol 11. Benzylation of an inexpensive starting material 1 (monohydrate) afforded $\mathbf{8},{ }^{29}$ ) which was smoothly brominated to give 9. On the other hand, the tert-butylation of $\mathbf{1}$ was accomplished using Ohta's system. ${ }^{30)}$ It was noted that although the yield of $\mathbf{1 0}^{31)}$ was quite low, this method was the best way for our intensive examination because the production of any undesired byproducts was suppressed and the unreacted $\mathbf{1}$ was easily removed. For the protection of the catechol moiety, the reaction of $\mathbf{1 0}$ with benzalchloride in pyridine was carried out to provide $\mathbf{1 1 .}$

The phenol 11 and bromide 9 were reacted by heating at $160^{\circ} \mathrm{C}$ to afford the biaryl ether 12 , which was successively deprotected without rigorous purification (Chart 5). Selective removal of the benzylidene group was achieved by use of the $\mathrm{SnCl}_{2}-\mathrm{EtSH}$ combination system ${ }^{32)}$ to give $\mathbf{1 3}$ in $76 \%$ yield. Compound 14 could be obtained by the reprotection of the catechol part of 13. Finally, the tert-butyl group of $\mathbf{1 4}$ was removed by $\mathrm{SnCl}_{2}$, which afforded the desired carboxylic acid 15. Thus, we succeeded in constructing the synthetic compo- nent of coriariin $\mathrm{B}$.

In conclusion, we investigated the formation of a highly functionalized biaryl ether derived from 1 via the classical Ullmann reaction. By using the benzylidene group, the biaryl ether 5d was sufficiently obtained, and dehydrodigallic acid (3) was readily synthesized. Additionally, we demonstrated the construction of the DHDG part of coriariin B. We believe that compound $\mathbf{1 5}$, which is derivative of $\mathbf{3}$, must be a common building block toward the synthesis of the ellagitannins.

\section{Experimental}

Melting points were measured using a Yanagimoto micro melting point hot-plate and are uncorrected. The IR spectra were recorded using a SHIMADZU FTIR-8400 spectrophotometer. The NMR spectra were obtained using a JEOL $\alpha-400$ $(400 \mathrm{MHz})$ or a JEOL JNX-ECX500 $(500 \mathrm{MHz})$ instrument. The chemical shifts are given in $\delta$ ppm with tetramethylsilane (TMS) as an internal standard. The elemental analyses were performed using a Thermo Scientific FlashEA1112 analyzer. FAB-MS was obtained using a JEOL JMS-AX505HAD instrument with $m$-nitrobenzyl alcohol as the matrix. Electron ionization-mass spectra (EI-MS) was obtained using a JMSGCmate II instrument. Silica gel column chromatography was carried out using a wakogel ${ }^{\circledR}$ C-200 (Wako). TLC analysis was performed on Kieselgel $60 \mathrm{~F}_{254}$ (Merck) plates. Copper dust was activated by the reported method. ${ }^{21)}$ Solvents were dried by the standard procedure.

General Procedure for Ullmann Reaction A mixture of $\mathrm{ArOH}(\mathbf{6 a}-\mathbf{e})(1.00 \mathrm{mmol}), \operatorname{ArBr}(7)(1.60 \mathrm{~g}, 3.00 \mathrm{mmol})$, 
and activated $\mathrm{Cu}(381 \mathrm{mg}, 6.00 \mathrm{mmol})$ in DMA $(2.5 \mathrm{~mL})$ was refluxed under $\mathrm{N}_{2}$ in a pre-heated oil bath at $200^{\circ} \mathrm{C}$. After stirring for $15-30 \mathrm{~min}$, the reaction mixture was cooled to ambient temperature, diluted with EtOAc, and filtered. The filtrate was poured into $\mathrm{H}_{2} \mathrm{O}$ and extracted with EtOAc. The combined organic layer was washed with brine, dried over $\mathrm{MgSO}_{4}$, and filtered. The filtrate was evaporated and the resulting residue was purified by silica gel column chromatography $(1: 4-1: 3$; EtOAc-hexane), providing the biaryl ether product $(\mathbf{5 a} \mathbf{a}-\mathbf{e})$.

Methyl 3,4,5-Tribenzyloxy-2-(2,3-dibenzyloxy-5-methoxycarbonylphenoxy)benzoate $^{10)} \quad$ (5a): From 6a (455 mg, $1.00 \mathrm{mmol}), \mathbf{5 a}(547 \mathrm{mg}, \mathrm{ca} .67 \%$, yellow oil) was obtained by the same procedure mentioned above. The reaction was carried out for $30 \mathrm{~min} ;{ }^{1} \mathrm{H}-\mathrm{NMR}\left(500 \mathrm{MHz}, \mathrm{CDCl}_{3}\right) \delta: 3.72(3 \mathrm{H}, \mathrm{s}$, $\mathrm{OMe}), 3.80$ (3H, s, OMe), $4.97\left(2 \mathrm{H}, \mathrm{s}, \mathrm{CH}_{2}\right), 5.13\left(4 \mathrm{H}, \mathrm{s}, \mathrm{CH}_{2}\right)$, $5.14\left(2 \mathrm{H}, \mathrm{s}, \mathrm{CH}_{2}\right), 5.15\left(2 \mathrm{H}, \mathrm{s}, \mathrm{CH}_{2}\right), 6.91(1 \mathrm{H}, \mathrm{d}, J=1.5 \overline{\mathrm{Hz}}$, $\mathrm{ArH}), 7.11-7.47(27 \mathrm{H}, \mathrm{m}, \mathrm{ArH}) ; \mathrm{IR}\left(\mathrm{CHCl}_{3}\right) v_{\max } 3033,3015$, 2953, 2357, 1717, 1539, 1435, 1338, 1224, 1206, 1093, 1011, $770,697,666 \mathrm{~cm}^{-1}$.

Methyl 3,4,5-Tribenzyloxy-2-(2,3-dimethoxy-5-methoxycarbonylphenoxy)benzoate (5b): From 6b (212 mg, $1.00 \mathrm{mmol}$ ), 5b $(527 \mathrm{mg}, 79 \%$, pale yellow solid) was obtained by the same procedure mentioned above. The reaction was carried out for $30 \mathrm{~min}$; mp $142-144^{\circ} \mathrm{C}$; ${ }^{1} \mathrm{H}-\mathrm{NMR}\left(500 \mathrm{MHz}, \mathrm{CDCl}_{3}\right)$ $\delta: 3.73(3 \mathrm{H}, \mathrm{s}, \mathrm{OMe}), 3.81(3 \mathrm{H}, \mathrm{s}, \mathrm{OMe}), 3.94(3 \mathrm{H}, \mathrm{s}, \mathrm{OMe})$, $3.94(3 \mathrm{H}, \mathrm{s}, \mathrm{OMe}), 4.96\left(2 \mathrm{H}, \mathrm{s}, \mathrm{CH}_{2}\right), 5.13\left(2 \mathrm{H}, \mathrm{s}, \mathrm{CH}_{2}\right), 5.14$ $\left(2 \mathrm{H}, \mathrm{s}, \mathrm{CH}_{2}\right), 6.84(1 \mathrm{H}, \mathrm{d}, J=1.5 \mathrm{~Hz}, \mathrm{ArH}), 7.10-7.48(17 \mathrm{H}, \mathrm{m}$,

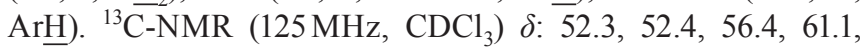
$71 . \overline{5}, 75.7,75.8,107.3,108.8,111.0,119.9,125.0,127.9,128.1$, $128.3,128.3,128.4,128.4,128.5,128.8,128.8,136.4,136.9$, $136.9,142.0,142.6,146.7,146.9,150.1,152.4,153.4,165.3$, 166.6; IR (KBr) $v_{\max } 2948,2360,2334,1721,1595,1467,1437$, 1417, 1337, 1224, 1097, 1012, 748, 697, 668, 420 $\mathrm{cm}^{-1}$; high resolution (HR)-MS (EI) Calcd for $\mathrm{C}_{39} \mathrm{H}_{36} \mathrm{O}_{10}[\mathrm{M}]^{+}$: 664.2308; Found: $664.2277[\mathrm{M}]^{+}$. Anal. Calcd for $\mathrm{C}_{39} \mathrm{H}_{36} \mathrm{O}_{10}: \mathrm{C}, 70.47 ; \mathrm{H}$, 5.46. Found: C, 70.74; H, 5.41.

Methyl 3,4,5-Tribenzyloxy-2-(2,3-ethoxymethylenedioxy-5methoxycarbonylphenoxy)benzoate (5c): From 6c (240 mg, $1.00 \mathrm{mmol}), 5 \mathbf{c}(494 \mathrm{mg}, 71 \%$, colorless oil) was obtained by the same procedure mentioned above. The reaction was carried out for $15 \mathrm{~min}$; ${ }^{1} \mathrm{H}-\mathrm{NMR}\left(500 \mathrm{MHz}\right.$, acetone- $\left.d_{6}\right) \delta$ : $1.19\left(3 \mathrm{H}, \mathrm{t}, J=6.5 \mathrm{~Hz}, \mathrm{OCH}_{2} \mathrm{CH}_{3}\right) 3.72(2 \mathrm{H}, \mathrm{q}, J=6.5 \mathrm{~Hz}$, $\left.\mathrm{OCH}_{2} \mathrm{CH}_{3}\right), 3.73(3 \mathrm{H}, \mathrm{s}, \mathrm{OMe}), 3.78(3 \mathrm{H}, \mathrm{s}, \mathrm{OMe}), 5.01(1 \mathrm{H}$, $\mathrm{d}, \overline{\mathrm{A}}$ of $\left.\mathrm{AB}, J=11.0 \mathrm{~Hz}, \underline{\mathrm{CH}}_{\mathrm{A}} \mathrm{H}_{\mathrm{B}}\right), 5.03(1 \mathrm{H}, \mathrm{d}, \mathrm{B}$ of $\mathrm{AB}$, $\left.J=11.0 \mathrm{~Hz}, \mathrm{CH}_{\mathrm{A}} \underline{\mathrm{H}}_{\mathrm{B}}\right), 5.21\left(2 \mathrm{H}, \mathrm{s}, \mathrm{CH}_{2}\right), 5.25\left(2 \mathrm{H}, \mathrm{s}, \mathrm{CH}_{2}\right), 7.03$ $(1 \mathrm{H}, \mathrm{d}, J=1.5 \mathrm{~Hz}, \mathrm{ArH}), 7.09(1 \mathrm{H}, \mathrm{s}), 7.48(1 \mathrm{H}, \mathrm{s}), 7.20-7.59$ $(16 \mathrm{H}, \mathrm{m}, \mathrm{ArH}) ;{ }^{13} \mathrm{C}-\mathrm{NMR}\left(125 \mathrm{MHz}\right.$, acetone- $\left.d_{6}\right) \delta: 15.1$, $52.4,52.4,60.3,71.8,76.0,76.2,103.9,111.3,112.5,120.8$, $121.3,124.9,128.7,128.8,128.8,128.9,129.0,129.1,129.3$, $129.4,137.6,137.9,138.0,138.6,142.7,142.8,147.3,147.3$, 148.5, 151.1, 165.3, 166.2; IR $\left(\mathrm{CHCl}_{3}\right) v_{\max } 3030,3015,2953$, $1714,1635,1438,1372,1313,1212,1085,769,697,666 \mathrm{~cm}^{-1}$; HR-MS (EI) Calcd for $\mathrm{C}_{40} \mathrm{H}_{36} \mathrm{O}_{11}[\mathrm{M}]^{+}$: 692.2258; Found: $692.2225[\mathrm{M}]^{+}$.

Methyl 2-(2,3-Benzaloxy-5-methoxycarbonylphenoxy)-3,4,5tribenzyloxybenzoate (5d): The mixture of $\mathbf{6 d}(2.72 \mathrm{~g}$, $10.0 \mathrm{mmol}), 7(16.0 \mathrm{~g}, 30.0 \mathrm{mmol})$, and activated $\mathrm{Cu}(3.81 \mathrm{~g}$, $60.0 \mathrm{mmol})$ in DMA $(25 \mathrm{~mL})$ was refluxed under $\mathrm{N}_{2}$ in a pre-heated oil bath at $200^{\circ} \mathrm{C}$. After stirring for $15 \mathrm{~min}$, the reaction mixture was cooled to ambient temperature, diluted with EtOAc $(400 \mathrm{~mL})$, and filtered using celite. The filtrate was poured into $\mathrm{H}_{2} \mathrm{O}(400 \mathrm{~mL})$ and extracted with EtOAc $(400 \mathrm{~mL} \times 3)$. The combined organic layer was washed with brine $(300 \mathrm{~mL})$, dried over $\mathrm{MgSO}_{4}$, and filtered. The filtrate was evaporated and the resulting residue $(17.6 \mathrm{~g})$ was purified by silica gel column chromatography (1:4; EtOAc-hexane), providing $5 \mathbf{d}(6.60 \mathrm{~g}, 91 \%)$ as a yellow oil; ${ }^{1} \mathrm{H}-\mathrm{NMR}(500 \mathrm{MHz}$, $\left.\mathrm{CDCl}_{3}\right) \delta: 3.74(3 \mathrm{H}, \mathrm{s}, \mathrm{OMe}), 3.81(3 \mathrm{H}, \mathrm{s}, \mathrm{OMe}), 4.96(2 \mathrm{H}, \mathrm{s}$, $\left.\mathrm{CH}_{2}\right), 5.09\left(2 \mathrm{H}, \mathrm{s}, \mathrm{CH}_{2}\right), 5.12\left(2 \mathrm{H}, \mathrm{s}, \mathrm{CH}_{2}\right), 6.95(1 \mathrm{H}, \mathrm{s}, \mathrm{PhCH})$, $7.03(1 \mathrm{H}, \mathrm{d}, J=2.0 \mathrm{~Hz}, \operatorname{Ar} \underline{\mathrm{H}}), 7.24(1 \mathrm{H}, \mathrm{d}, J=2.0 \mathrm{~Hz}, \operatorname{Ar} \underline{\mathrm{H}})$, $7.13-7.53(21 \mathrm{H}, \mathrm{m}, \mathrm{ArH}) ;{ }^{13} \overline{\mathrm{C}}-\mathrm{NMR}\left(125 \mathrm{MHz}, \mathrm{CDCl}_{3}\right) \delta: 52.2$, $52.4,71.5,75.7,75.8,104.3,110.9,112.0,112.5,119.9,124.3$, $126.5,127.9,128.0,128.2,128.3,128.4,128.4,128.7,128.8$, $130.5,135.6,136.4,136.9,137.0,139.7,142.1,142.8,146.7$, 146.8, 149.3, 150.2, 165.3, 166.3; IR $\left(\mathrm{CHCl}_{3}\right) v_{\max } 3030,3011$, 2953, 1712, 1635, 1499, 1437, 1363, 1226, 1201, 1087, 1018, 909, 747, $697 \mathrm{~cm}^{-1}$; HR-MS (EI) Calcd for $\mathrm{C}_{44} \mathrm{H}_{36} \mathrm{O}_{10}[\mathrm{M}]^{+}$: 724.2308; Found: $724.2283\left[\mathrm{M}^{+}\right.$.

Methyl 3,4,5-Tribenzyloxy-2-(2,3-p-methoxybenzaloxy-5methoxycarbonylphenoxy)benzoate (5e): From 6e (302 mg, $1.00 \mathrm{mmol})$, 5e (346 mg, 46\%, yellow amorphous) was obtained by the same procedure mentioned above. The reaction was carried out for $15 \mathrm{~min}$; ${ }^{1} \mathrm{H}-\mathrm{NMR}\left(400 \mathrm{MHz}, \mathrm{DMSO}-d_{6}\right) \delta$ : $3.70(3 \mathrm{H}, \mathrm{s}, \mathrm{OMe}), 3.75$ (3H, s, OMe), 3.77 (3H, s, OMe), 4.85 $\left(1 \mathrm{H}, \mathrm{d}, \mathrm{A}\right.$ of $\left.\mathrm{AB}, J=10.8 \mathrm{~Hz}, \mathrm{CH}_{\mathrm{A}} \mathrm{H}_{\mathrm{B}}\right), 4.92(1 \mathrm{H}, \mathrm{d}, \mathrm{B}$ of $\mathrm{AB}$, $\left.J=10.8 \mathrm{~Hz}, \mathrm{CH}_{\mathrm{A}} \underline{\mathrm{H}}_{\mathrm{B}}\right), 5.09\left(2 \mathrm{H}, \mathrm{s}, \mathrm{CH}_{2}\right), 5.21\left(2 \mathrm{H}, \mathrm{s}, \mathrm{CH}_{2}\right), 6.82$ $(1 \mathrm{H}, \mathrm{s}, \mathrm{PhC} \underline{\mathrm{H}}), 6.98-7.51(22 \mathrm{H}, \mathrm{m}, \overline{\mathrm{Ar}} \underline{\mathrm{H}}) ;{ }^{13} \mathrm{C}-\mathrm{NMR}(125 \mathrm{MHz}$, acetone- $\left.d_{6}\right) \delta: 52.3,52.4,55.6,71.4,75.9,76.1,104.1,111.2$, $112.8,112.9,114.8,120.7,124.8,128.5,128.7,128.7,128.7$, $128.8,128.9,129.0,129.0,129.3,137.5,137.9,137.9,140.4$, $142.9,142.9,147.2,147.2,150.3,150.9,162.3,165.3,166.2$; IR $\left(\mathrm{CHCl}_{3}\right) v_{\max } 3031,3013,2953,2357,1714,1635,1499,1436$, 1306, 1252, 1223, 1214, 1172, 1086, 1024, 773, $668 \mathrm{~cm}^{-1}$; HR-MS (EI) Calcd for $\mathrm{C}_{45} \mathrm{H}_{38} \mathrm{O}_{11}[\mathrm{M}]^{+}$: 754.2415; Found: $754.2378[\mathrm{M}]^{+}$.

Conversion of $5 d$ to 5 a To a solution of $5 d$ d $(643 \mathrm{mg}$, $0.887 \mathrm{mmol})$ in $\mathrm{MeOH}(8 \mathrm{~mL})$ and EtOAc $(8 \mathrm{~mL}), 10 \% \mathrm{Pd}-\mathrm{C}$ $(64 \mathrm{mg})$ was added at room temperature. After stirring for 30 min under $\mathrm{H}_{2}$, the reaction mixture was filtered and concentrated in vacuo to give a yellow amorphous (371 mg) powder, which was used in the next reaction without further purification. A mixture of the above residue $(371 \mathrm{mg})$ and $\mathrm{K}_{2} \mathrm{CO}_{3}(1.23 \mathrm{~g}, 8.90 \mathrm{mmol})$ in DMF $(20 \mathrm{~mL})$ was stirred at room temperature. $\mathrm{BnBr}(1.06 \mathrm{~mL}, 8.92 \mathrm{~mol})$ was added to the solution and the mixture was heated at $80^{\circ} \mathrm{C}$ with stirring. After $1 \mathrm{~h}$, the reaction mixture was poured into $\mathrm{H}_{2} \mathrm{O}(100 \mathrm{~mL})$ and extracted with EtOAc $(50 \mathrm{~mL} \times 3)$. The combined organic layer was washed with brine $(50 \mathrm{~mL})$, dried over $\mathrm{MgSO}_{4}$, and filtered. The filtrate was evaporated and the resulting residue $(1.63 \mathrm{~g})$ was purified by silica gel column chromatography (1:4; EtOAc-hexane), providing 5a (661 mg, 91\%) as a pale yellow solid.

Dehydrodigallic Acid (3) To a solution of 5d (1.45 g, $2.00 \mathrm{mmol})$ in THF $(10 \mathrm{~mL}), 10 \% \mathrm{KOH}$ aq. $(10 \mathrm{~mL})$ was added and the mixture was stirred at room temperature. After $18 \mathrm{~h}, \mathrm{MeOH}(5 \mathrm{~mL})$ was added and the mixture was heated at $60^{\circ} \mathrm{C}$ with stirring for $24 \mathrm{~h}$. The reaction mixture was quenched with $10 \% \mathrm{HCl}$ aq. $(10 \mathrm{~mL})$ and extracted with EtOAc $(50 \mathrm{~mL} \times 3)$. The combined organic layer was washed with brine $(50 \mathrm{~mL})$, dried over $\mathrm{MgSO}_{4}$, and filtered. The filtrate was evaporated and the obtained pale yellow solid $(1.45 \mathrm{~g})$ 
was recrystallized from EtOAc-hexane, providing the dicarboxylic acid $(1.29 \mathrm{~g}, 93 \%)$ as a colorless solid; $\mathrm{mp} 215-217^{\circ} \mathrm{C}$ (EtOAc-hexane); ${ }^{1} \mathrm{H}-\mathrm{NMR} \quad\left(500 \mathrm{MHz}\right.$, acetone- $\left.d_{6}\right) \quad \delta: 4.99$ $\left(1 \mathrm{H}, \mathrm{d}, \mathrm{A}\right.$ of $\left.\mathrm{AB}, J=11.0 \mathrm{~Hz}, \mathrm{CH}_{\mathrm{A}} \mathrm{H}_{\mathrm{B}}\right), 5.03(1 \mathrm{H}, \mathrm{d}, \mathrm{B}$ of $\mathrm{AB}$, $\left.J=11.0 \mathrm{~Hz}, \mathrm{CH}_{\mathrm{A}} \underline{\mathrm{H}}_{\mathrm{B}}\right), 5.18\left(2 \mathrm{H}, \mathrm{s}, \mathrm{CH}_{2}\right), 5.26\left(2 \mathrm{H}, \mathrm{s}, \mathrm{CH}_{2}\right), 7.08$ $(1 \mathrm{H}, \mathrm{d}, J=1.0 \mathrm{~Hz}, \mathrm{ArH}), 7.13(1 \mathrm{H}, \mathrm{s}, \mathrm{PhCH}), 7.19-7.61(22 \mathrm{H}$, $\mathrm{m}, \mathrm{ArH}) ;{ }^{13} \mathrm{C}-\mathrm{NMR}\left(125 \mathrm{MHz}\right.$, acetone- $\left.d_{6}\right) \delta: 71.8,75.9,76.1$, $104.3,111.6,112.7,113.1,121.2,125.3,127.3,128.7,128.8$, $128.8,128.9,129.0,129.1,129.3,129.4,129.5,131.3,136.7$, $137.7,138.0,138.0,140.4,143.1,147.2,147.3,150.1,151.0$, 165.7, 166.7; IR (KBr) $v_{\max } 3033,2945,2362,1685,1631,1498$, 1438, 1367, 1305, 1218, 1089, 1016, 737, $695 \mathrm{~cm}^{-1}$; HR-MS (EI) Calcd for $\mathrm{C}_{42} \mathrm{H}_{32} \mathrm{O}_{10}[\mathrm{M}]^{+}:$696.1995; Found: 696.2034 $[\mathrm{M}]^{+}$. The resulting product $(139 \mathrm{mg}, 0.200 \mathrm{mmol})$ was dissolved in $\mathrm{MeOH}(6 \mathrm{~mL}), 10 \% \mathrm{Pd}-\mathrm{C}(14.0 \mathrm{mg})$ was added to the solution and the mixture was stirred at room temperature under $\mathrm{H}_{2}$. After $2 \mathrm{~h}$, the reaction mixture was filtered and concentrated in vacuo, providing $3(66.7 \mathrm{mg}, 99 \%)$ as a brown solid; ${ }^{1} \mathrm{H}-\mathrm{NMR}\left(500 \mathrm{MHz}, \mathrm{DMSO}-d_{6}\right) \delta$ : $6.46(1 \mathrm{H}, \mathrm{d}$, $J=1.5 \mathrm{~Hz}, \mathrm{ArH}), 6.90(1 \mathrm{H}, \mathrm{s}, \operatorname{ArH}), 7.00(1 \mathrm{H}, \mathrm{d}, J=1.5 \mathrm{~Hz}$, $\mathrm{ArH}) ;{ }^{13} \mathrm{C}-\mathrm{NMR}\left(125 \mathrm{MHz}, \mathrm{DMSO}-d_{6}\right) \delta: 106.6,108.2,110.5$, $115.2,119.9,136.0,139.0,139.2,139.7,142.5,145.6,147.5$, 166.3 , 167.5; IR (KBr) $v_{\max } 3498,2357,2342,1685,1611,1522$, 1440, 1329, 1244, 1190, 1101, 1034, $668 \mathrm{~cm}^{-1}$; HR-MS (EI) Calcd for $\mathrm{C}_{14} \mathrm{H}_{10} \mathrm{O}_{10}[\mathrm{M}]^{+}$: 338.0274; Found: $338.0289[\mathrm{M}]^{+}$. The spectral data of the synthetic 3 matched the data provided in the literature. ${ }^{26)}$

Benzyl 3,4,5-Tribenzyloxybenzoate ${ }^{29)}$ (8) A mixture of gallic acid monohydrate (1) $(100 \mathrm{~g}, 0.532 \mathrm{~mol}), \mathrm{K}_{2} \mathrm{CO}_{3}(587 \mathrm{~g}$, $4.25 \mathrm{~mol})$, and ${ }^{n} \mathrm{Bu}_{4} \mathrm{NI}(9.9 \mathrm{~g}, 26.8 \mathrm{mmol})$ in DMF $(1 \mathrm{~L})$ was stirred using a mechanical stirrer at room temperature. $\mathrm{BnCl}$ $(501 \mathrm{~mL}, 4.25 \mathrm{~mol})$ was added to the suspention and the mixture was heated at $100^{\circ} \mathrm{C}$ with stirring for $4.5 \mathrm{~h}$. The reaction mixture was cooled to ambient temperature, poured into the mixed solution of $\mathrm{H}_{2} \mathrm{O}(5 \mathrm{~L})$ and hexane $(0.5 \mathrm{~L})$, and stirred. The precipitate was then collected on a glass filter and washed with $\mathrm{H}_{2} \mathrm{O}$ and hexane. The obtained white solid (497.2 g) was recrystallized from acetone, providing 8 (243 g, 86\%) as colorless needles; mp $101.3-102.8^{\circ} \mathrm{C}$ (acetone); ${ }^{1} \mathrm{H}-\mathrm{NMR}(500 \mathrm{MHz}$, $\left.\mathrm{CDCl}_{3}\right) \delta: 5.10\left(4 \mathrm{H}, \mathrm{s}, \mathrm{CH}_{2}\right), 5.15\left(2 \mathrm{H}, \mathrm{s}, \mathrm{CH}_{2}\right), 5.33(2 \mathrm{H}, \mathrm{s}$, $\left.\mathrm{CH}_{2}\right), 7.26-7.44(22 \mathrm{H}, \mathrm{m}, \mathrm{ArH}) ;{ }^{13} \mathrm{C}-\mathrm{NMR}\left(125 \mathrm{MHz}, \mathrm{CDCl}_{3}\right)$ $\delta$ 66.8, 71.2, 75.1, 109.2, 125.2, 127.6, 128.0, 128.0, 128.1, $128.2,128.2,128.6,128.6,136.1,136.7,137.5,142.5,152.6$, 165.9; IR (KBr) $v_{\max } 3064,3032,2945,2866,1710,1594,1500$, 1454, 1428, 1385, 1366, 1338, 1204, 1128, 1029, 971, 862, 753, $730,695,604 \mathrm{~cm}^{-1}$; HR-MS (EI) Calcd for $\mathrm{C}_{35} \mathrm{H}_{30} \mathrm{O}_{5}[\mathrm{M}]^{+}$: 530.2093; Found: $530.2115\left[\mathrm{M}^{+}\right.$.

Benzyl 3,4,5-Tribenzyloxy-2-bromobenzoate (9) То a stirring solution of $8(79.6 \mathrm{~g}, 150 \mathrm{mmol})$ in DMF $(350 \mathrm{~mL})$, a solution of $N$-bromosuccinimide (NBS) $(30.7 \mathrm{~g}, 172.5 \mathrm{mmol})$ in DMF $(70 \mathrm{~mL})$ was dropwise added at room temperature. After stirring for $24 \mathrm{~h}, \mathrm{H}_{2} \mathrm{O}$ was added to the solution and the resulting mixture was then stirred. The precipitate was collected on a glass filter and washed with $\mathrm{H}_{2} \mathrm{O}$ and hexane. The obtained solid (112.4 g) was recrystallized from acetone, providing 9 (78.5 g, 86\%) as colorless needles; mp $123.8-124.4^{\circ} \mathrm{C}$ (acetone); ${ }^{1} \mathrm{H}-\mathrm{NMR}\left(500 \mathrm{MHz}, \mathrm{CDCl}_{3}\right) \delta: 5.03\left(2 \mathrm{H}, \mathrm{s}, \mathrm{CH}_{2}\right)$, $5.10\left(4 \mathrm{H}, \mathrm{s}, \mathrm{CH}_{2}\right), 5.37\left(2 \mathrm{H}, \mathrm{s}, \mathrm{CH}_{2}\right), 7.29-7.51(21 \mathrm{H}, \mathrm{m}, \mathrm{ArH})$; ${ }^{13} \mathrm{C}-\mathrm{NMR}\left(125 \mathrm{MHz}, \mathrm{CDCl}_{3}\right) \delta: 67.6,71.4,75.6,75.9,110.6$, $112.4,127.7,128.4,128.4,128.5,128.5,128.6,128.6,128.8$, $128.8,128.8,135.6,136.1,136.7,136.9,146.0,151.1,151.8$,
165.9; IR (KBr) $v_{\max } 3030,2362,2333,1700,1577,1454,1369$, 1329, 1216, 1159, 1100, 955, 909, 740, 698 $\mathrm{cm}^{-1}$; HR-MS (EI) Calcd for $\mathrm{C}_{35} \mathrm{H}_{29} \mathrm{O}_{5} \mathrm{Br}[\mathrm{M}]^{+}$: 608.1198; Found: $608.1241[\mathrm{M}]^{+}$. Anal. Calcd for $\mathrm{C}_{35} \mathrm{H}_{29} \mathrm{O}_{5} \mathrm{Br}: \mathrm{C}, 68.97 ; \mathrm{H}, 4.80$. Found: C, 68.74; H, 4.75 .

tert-Butyl Gallate ${ }^{31)}$ (10) A mixture of gallic acid monohydrate (1) $(47.0 \mathrm{~g}, 250 \mathrm{mmol}), t-\mathrm{BuOH}(300 \mathrm{~mL})$, and $t$-BuOAc $(300 \mathrm{~mL})$ was stirred at $0^{\circ} \mathrm{C}$ under $\mathrm{N}_{2}$. Conc. $\mathrm{H}_{2} \mathrm{SO}_{4}(25 \mathrm{~mL})$ was carefully added to the solution with maintaining the temperature at $0^{\circ} \mathrm{C}$. After stirring for $1 \mathrm{~h}$ at the same temperature, the resulting mixture was warmed to room temperature and stirred for $5 \mathrm{~d}$. The reaction mixture was quenched with sat. $\mathrm{NaHCO}_{3}$ aq., poured into $\mathrm{H}_{2} \mathrm{O}(500 \mathrm{~mL})$, and extracted with $\mathrm{Et}_{2} \mathrm{O}(500 \mathrm{~mL} \times 3)$. The combined organic layer was washed with brine $(300 \mathrm{~mL})$, dried over $\mathrm{MgSO}_{4}$, and filtered. The filtrate was evaporated and the resulting yellow residue $(64.5 \mathrm{~g})$ was dissolved in $\mathrm{CHCl}_{3}(100 \mathrm{~mL})$. After removal of the insoluble precipitate by filtration, the filtrate was concentrated in vacuo and the yellow residue $(29.5 \mathrm{~g})$ was separated by silica gel column chromatography (1:4; EtOAc-hexane). The obtained pale yellow solid $(24.5 \mathrm{~g})$ was recrystallized from $\mathrm{CHCl}_{3}$, providing $\mathbf{1 0}(21.7 \mathrm{~g}, 38 \%)$ as a colorless solid. Analytical sample of $\mathbf{1 0}$ was obtained by further recrystallization from $\mathrm{CHCl}_{3}$-hexane; mp $146-147^{\circ} \mathrm{C}\left(\mathrm{CHCl}_{3}\right.$-hexane); IR $(\mathrm{KBr}) v_{\max } 3377,3314,3227,2980,1697,1670,1624,1445$, 1371, 1348, 1256, 1157, 1026, $771 \mathrm{~cm}^{-1}$; ${ }^{1} \mathrm{H}-\mathrm{NMR}(500 \mathrm{MHz}$, acetone- $\left.d_{6}\right) \quad \delta: 1.53(9 \mathrm{H}, \mathrm{s}, \mathrm{O} t-\mathrm{Bu}), 7.07(2 \mathrm{H}, \mathrm{s}, \mathrm{Ar} \underline{\mathrm{H}})$; ${ }^{13} \mathrm{C}-\mathrm{NMR}\left(125 \mathrm{MHz}\right.$, acetone- $\left.d_{6}\right) \delta: 28.3,80.3,109.7,123.6$, 138.2, 145.8, 166.0; Anal. Calcd for $\mathrm{C}_{11} \mathrm{H}_{14} \mathrm{O}_{5}: \mathrm{C}, 58.40 ; \mathrm{H}$, 6.24. Found: C, 58.35; H, 6.40.

tert-Butyl 3,4-Benzaloxy-5-hydroxybenzoate (11) To a solution of $10(10.0 \mathrm{~g}, 44.2 \mathrm{mmol})$ in pyridine $(25.0 \mathrm{~mL})$, benzal chloride $(8.54 \mathrm{~mL}, 66.3 \mathrm{mmol})$ was added and the mixture was heated at $110^{\circ} \mathrm{C}$ under $\mathrm{N}_{2}$. After stirring for $8 \mathrm{~h}$, toluene was added to the reaction mixture and the solvent was removed in vacuo. The resulting purple residue was dissolved in $\mathrm{CHCl}_{3}$ and filtered with $\mathrm{SiO}_{2}$ pad. After concentration of the filtrate, pale yellow solid $(16.2 \mathrm{~g})$ was recrystallized from $\mathrm{CHCl}_{3}$-hexane, providing $11(9.71 \mathrm{~g}, 70 \%)$ as a colorless solid; mp $167.5-169.8^{\circ} \mathrm{C}\left(\mathrm{CHCl}_{3}\right.$-hexane); IR (KBr) v $v_{\max } 3369$, 2976, 1684, 1645, 1614, 1512, 1447, 1398, 1337, 1335, 1305, 1259, 1219, 1163, 1076, 1076, 1016, 766, 696 $\mathrm{cm}^{-1}$; ${ }^{1} \mathrm{H}-\mathrm{NMR}$ $\left(400 \mathrm{MHz}, \mathrm{CDCl}_{3}\right) \delta: 1.57(9 \mathrm{H}, \mathrm{s}, \mathrm{O} t-\mathrm{Bu}), 7.04(1 \mathrm{H}, \mathrm{s}, \mathrm{PhCH})$, $7.13(1 \mathrm{H}, \mathrm{d}, J=1.6 \mathrm{~Hz}, \operatorname{ArH}), 7.33(1 \mathrm{H}, \mathrm{d}, J=1.6 \mathrm{~Hz}, \operatorname{Ar} \underline{\mathrm{H}})$, 7.44-7.57 (5H, m, ArH $) ;{ }^{13} \mathrm{C}-\mathrm{NMR}\left(125 \mathrm{MHz}\right.$, acetone- $\left.d_{6}\right) \delta$ : 28.2, 81.0, 102.4, 111.9, 114.5, 127.1, 127.2, 129.5, 131.2, 137.0, 139.0, 140.9, 149.7, 165.3; Anal. Calcd for $\mathrm{C}_{18} \mathrm{H}_{18} \mathrm{O}_{5}$ : C, 68.78; H, 5.77. Found: C, 68.50; H, 5.86.

Benzyl 2-(2,3-Benzaloxy-5-tert-butoxycarbonylphenoxy)3,4,5-tribenzyloxybenzoate (12) Two round-bottomed flasks $(200 \mathrm{~mL})$ were equipped with 11 (3.15 g, $10.0 \mathrm{mmol})$, $9(18.3 \mathrm{~g}, 30.0 \mathrm{mmol})$, activated $\mathrm{Cu}(3.81 \mathrm{~g}, 60.0 \mathrm{mmol})$, and DMA $(25 \mathrm{~mL})$, respectively. The each mixture was heated at $160^{\circ} \mathrm{C}$ under $\mathrm{N}_{2}$. After stirring for $30 \mathrm{~min}$, the each reaction mixture was cooled to ambient temperature, diluted with EtOAc $(250 \mathrm{~mL})$, and filtered. The filtrate were combined, poured into $\mathrm{H}_{2} \mathrm{O}(500 \mathrm{~mL})$, and extracted with EtOAc $(500 \mathrm{~mL} \times 3)$. The combined organic layer was washed with brine $(300 \mathrm{~mL})$, dried over $\mathrm{MgSO}_{4}$, and filtered. The filtrate was evaporated and the resulting residue $(46.3 \mathrm{~g})$ was purified by silica gel column chromatography (1:4; EtOAc-hexane) to 
give a colorless residue $(39.9 \mathrm{~g})$ as a mixture containing $\mathbf{1 2}$, which was used in the next reaction without further purification. Analytical sample of $\mathbf{1 2}$ was separately obtained by silica gel column chromatography as a colorless oil; ${ }^{1} \mathrm{H}-\mathrm{NMR}$ $\left(500 \mathrm{MHz}\right.$, acetone- $\left.d_{6}\right) \delta: 1.52(9 \mathrm{H}, \mathrm{s}, \mathrm{O} t-\mathrm{Bu}), 4.98(1 \mathrm{H}, \mathrm{d}, \mathrm{A}$ of $\left.\mathrm{AB}, J=10.5 \mathrm{~Hz}, \mathrm{CH}_{\mathrm{A}} \mathrm{H}_{\mathrm{B}}\right), 5.01(1 \mathrm{H}, \mathrm{d}, \mathrm{B}$ of $\mathrm{AB}, J=10.5 \mathrm{~Hz}$, $\left.\mathrm{CH}_{\mathrm{A}} \mathrm{H}_{\mathrm{B}}\right), 5.17\left(2 \mathrm{H}, \mathrm{s}, \mathrm{CH}_{2}\right), 5.19(1 \mathrm{H}, \mathrm{d}, \mathrm{A}$ of $\mathrm{AB}, J=12.5 \mathrm{~Hz}$, $\left.\mathrm{CH}_{\mathrm{A}} \mathrm{H}_{\mathrm{B}}\right), 5.24\left(1 \mathrm{H}, \mathrm{d}, \mathrm{B}\right.$ of $\left.\mathrm{AB}, J=12.5 \mathrm{~Hz}, \mathrm{CH}_{\mathrm{A}} \underline{\mathrm{H}}_{\mathrm{B}}\right), 5.25(2 \mathrm{H}$, s, $\left.\mathrm{CH}_{2}\right), 6.98(1 \mathrm{H}, \mathrm{d}, J=1.5 \mathrm{~Hz}, \mathrm{ArH}), 7.04(1 \mathrm{H}, \mathrm{s}, \mathrm{PhCH}), 7.10$ $(1 \mathrm{H}, \mathrm{d}, J=1.5 \mathrm{~Hz}, \operatorname{Ar} \underline{\mathrm{H}}), 7.50(1 \mathrm{H}, \mathrm{s}, \mathrm{ArH}), 7.19-7.56(25 \mathrm{H}$, $\mathrm{m}, \mathrm{ArH}) ;{ }^{13} \mathrm{C}-\mathrm{NMR}\left(125 \mathrm{MHz}\right.$, acetone- $\left.d_{6}\right) \delta: 28.2,67.4$, $71.8,76.0,76.2,81.2,104.0,111.5,112.5,112.7,120.9,126.9$, $127.2,128.6,128.8,128.9,129.0,129.1,129.3,129.5,131.2$, $136.7,136.8,137.5,137.8,137.9,139.7,142.8,142.8,147.3$, 147.4, 150.0, 151.0, 164.9, 165.0; IR $\left(\mathrm{CHCl}_{3}\right) v_{\max } 3034,3013$, 2490, 1704, 1635, 1498, 1436, 1367, 1217, 1159, 1087, 1017, 770, $697 \mathrm{~cm}^{-1}$; HR-MS (FAB, positive ion mode) Calcd for $\mathrm{C}_{53} \mathrm{H}_{47} \mathrm{O}_{10}[\mathrm{M}+\mathrm{H}]^{+}:$843.3169; Found: $843.3148[\mathrm{M}+\mathrm{H}]^{+}$.

Benzyl 3,4,5-Tribenzyloxy-2-(5-tert-butoxycarbonyl-2,3dihydroxyphenoxy)benzoate (13) To a stirring solution of the above residue $(39.9 \mathrm{~g})$ in $\mathrm{CH}_{2} \mathrm{Cl}_{2}(500 \mathrm{~mL}), \mathrm{EtSH}(14.8 \mathrm{~mL}$, $200 \mathrm{mmol})$ and $\mathrm{SnCl}_{2}(9.13 \mathrm{~g}, 50.0 \mathrm{mmol})$ were added at $0{ }^{\circ} \mathrm{C}$. After stirring for $22 \mathrm{~h}$ at room temperature, the reaction mixture was filtered and concentrated in vacuo. The resulting residue $(52.7 \mathrm{~g})$ was purified by silica gel column chromatography ( $1: 3-1: 2$; EtOAc-hexane), providing 13 (11.4 g, 76\%) as a colorless amorphous powder; ${ }^{1} \mathrm{H}-\mathrm{NMR}\left(500 \mathrm{MHz}\right.$, acetone- $\left.d_{6}\right) \delta$ : $1.46(9 \mathrm{H}, \mathrm{s}, \mathrm{Ot}-\mathrm{Bu}), 4.99\left(2 \mathrm{H}, \mathrm{s}, \mathrm{CH}_{2}\right), 5.20\left(2 \mathrm{H}, \mathrm{s}, \mathrm{CH}_{2}\right), 5.21$ $\left(2 \mathrm{H}, \mathrm{s}, \mathrm{CH}_{2}\right), 5.25\left(2 \mathrm{H}, \mathrm{s}, \mathrm{CH}_{2}\right), 6.75(1 \mathrm{H}, \mathrm{d}, J=1.5 \mathrm{~Hz}, \mathrm{ArH})$, $7.18(1 \mathrm{H}, \mathrm{d}, J=1.5 \mathrm{~Hz}, \operatorname{ArH}), 7.49(1 \mathrm{H}, \mathrm{s}, \operatorname{ArH}), 7.23-7.56$ $(20 \mathrm{H}, \mathrm{m}, \mathrm{ArH}) ;{ }^{13} \mathrm{C}-\mathrm{NMR}\left(125 \mathrm{MHz}\right.$, acetone- $\left.d_{6}\right) \delta: 28.2,67.3$, $71.8,76.0,76.2,80.5,107.9,111.6,111.8,121.0,123.2,128.6$, $128.7,128.9,128.9,129.1,129.1,129.1,129.3,129.3,136.9$, $137.6,137.9,138.0,139.3,143.4,146.3,147.5,147.6,147.9$, $150.8,165.1,165.6 ;$ IR $\left(\mathrm{CHCl}_{3}\right) v_{\max } 3551,3034,3014,1703$, $1615,1455,1436,1367,1339,1311,1207,1178,11186,1068$, 966, 788, $697 \mathrm{~cm}^{-1}$; HR-MS (EI) Calcd for $\mathrm{C}_{46} \mathrm{H}_{42} \mathrm{O}_{10}[\mathrm{M}]^{+}$: 754.2778; Found: $754.2744[\mathrm{M}]^{+}$.

Benzyl 3,4,5-Tribenzyloxy-2-(2,3-dibenzyloxy-5-tert-butoxycarbonylphenoxy)benzoate (14) To a stirring solution of $13(1.51 \mathrm{~g}, 2.00 \mathrm{mmol})$ and $\mathrm{K}_{2} \mathrm{CO}_{3}(691 \mathrm{mg}, 5.00 \mathrm{mmol})$ in DMF $(10 \mathrm{~mL}), \mathrm{BnBr}(0.52 \mathrm{~mL}, 4.38 \mathrm{mmol})$ was added and the mixture was heated at $40^{\circ} \mathrm{C}$. After $2 \mathrm{~h}$, the reaction mixture was cooled to ambient temperature, poured into $\mathrm{H}_{2} \mathrm{O}(100 \mathrm{~mL})$, and extracted with EtOAc $(50 \mathrm{~mL} \times 3)$. The combined organic layer was washed with brine $(50 \mathrm{~mL})$, dried over $\mathrm{MgSO}_{4}$, and filtered. The filtrate was evaporated and the resulting yellow residue $(2.20 \mathrm{~g})$ was purified by silica gel column chromatography (1:4; EtOAc-hexane), providing 14 (1.71 g, 91\%) as a colorless solid. Analytical sample of $\mathbf{1 4}$ was obtained by recrystallization from acetone; $\mathrm{mp} 129-131^{\circ} \mathrm{C}$ (acetone); ${ }^{1} \mathrm{H}-\mathrm{NMR}\left(500 \mathrm{MHz}\right.$, acetone- $\left.d_{6}\right) \delta: 1.49(9 \mathrm{H}, \mathrm{s}, \mathrm{O} t-\mathrm{Bu}), 4.97$ $\left(2 \mathrm{H}, \mathrm{s}, \mathrm{CH}_{2}\right), 5.01\left(2 \mathrm{H}, \mathrm{s}, \mathrm{CH}_{2}\right), 5.20\left(4 \mathrm{H}, \mathrm{s}, \mathrm{CH}_{2}\right), 5.22(2 \mathrm{H}$, $\left.\mathrm{s}, \mathrm{CH}_{2}\right), 5.27\left(2 \mathrm{H}, \mathrm{s}, \mathrm{CH}_{2}\right), 6.86(1 \mathrm{H}, \mathrm{d}, J=2.0 \mathrm{~Hz}, \mathrm{ArH})$, 7.17-7.57 (32H, m, ArH $) ;{ }^{13} \mathrm{C}-\mathrm{NMR}\left(125 \mathrm{MHz}\right.$, acetone- $\left.d_{6}\right) \delta$ : 28.2, 67.4, 71.7, 71.8, 75.3, 76.0, 76.1, 81.2, 109.5, 109.6, 111.6, $121.0,127.8,128.4,128.5,128.6,128.7,128.7,128.8,128.9$, $129.0,129.1,129.2,129.3,136.8,137.6,137.8,138.0,138.0$, $139.0,142.1,142.9,147.2,147.6,150.8,153.5,165.0,165.2$; IR $(\mathrm{KBr}) v_{\max } 3031,2952,2357,1716,1594,1423,1371,1343$, 1222, 1095, 1000, 967, 859, 753, $697 \mathrm{~cm}^{-1}$; HR-MS (FAB, posi- tive ion mode) Calcd for $\mathrm{C}_{60} \mathrm{H}_{55} \mathrm{O}_{10}[\mathrm{M}+\mathrm{H}]^{+}$: 935.3795; Found: 935.3816 $[\mathrm{M}+\mathrm{H}]^{+}$. Anal. Calcd for $\mathrm{C}_{60} \mathrm{H}_{54} \mathrm{O}_{10}: \mathrm{C}, 77.07 ; \mathrm{H}$, 5.82. Found: C, 76.87; H, 5.87.

3,4-Dibenzyloxy-5-(2,3,4-tribenzyloxy-6-benzyloxycarbonylphenoxy)benzoic Acid (15) To a stirring solution of $14(935 \mathrm{mg}, 1.00 \mathrm{mmol})$ in $\mathrm{CH}_{2} \mathrm{Cl}_{2}(10 \mathrm{~mL}), \mathrm{AcOH}(286 \mu \mathrm{L}$, $5.00 \mathrm{mmol})$ and $\mathrm{SnCl}_{2}(457 \mathrm{mg}, 2.50 \mathrm{mmol})$ were subsequently added. After stirring for $22 \mathrm{~h}$ at room temperature, $\mathrm{AcOH}$ $(286 \mu \mathrm{L}, 5.00 \mathrm{mmol})$ and $\mathrm{SnCl}_{2}(457 \mathrm{mg}, 2.50 \mathrm{mmol})$ were added to the mixture and the resulting mixture was stirred. After $32 \mathrm{~h}$, the reaction mixture was filtered and concentrated in vacuo. The obtained yellow amorphous $(1.02 \mathrm{~g})$ was purified by silica gel column chromatography (1:1; EtOAc-hexane), providing $15(777 \mathrm{mg}, 88 \%)$ as a colorless solid. Analytical sample of $\mathbf{1 5}$ was obtained by recrystallization from acetonehexane; mp $137-139^{\circ} \mathrm{C}$ (acetone-hexane); ${ }^{1} \mathrm{H}-\mathrm{NMR}(500 \mathrm{MHz}$, acetone- $\left.d_{6}\right) \delta: 4.93\left(2 \mathrm{H}, \mathrm{s}, \mathrm{CH}_{2}\right), 5.01\left(2 \mathrm{H}, \mathrm{s}, \mathrm{CH}_{2}\right), 5.21(2 \mathrm{H}$, $\left.\mathrm{s}, \mathrm{CH}_{2}\right), 5.22\left(2 \mathrm{H}, \mathrm{s}, \mathrm{CH}_{2}\right), 5.25\left(2 \mathrm{H}, \mathrm{s}, \mathrm{CH}_{2}\right), 5.26(2 \mathrm{H}, \mathrm{s}$, $\left.\mathrm{CH}_{2}\right), 6.94(1 \mathrm{H}, \mathrm{d}, J=2.0 \mathrm{~Hz}, \mathrm{ArH}), 7.16-7.58$ (32H, m, ArH); ${ }^{13} \mathrm{C}$-NMR $\left(125 \mathrm{MHz}\right.$, acetone- $\left.d_{6}\right) \delta: 67.4,71.5,71.6,75.3,75.8$, $76.0,109.7,111.4,120.8,126.0,128.3,128.5,128.6,128.6$, $128.8,128.8,128.9,129.0,129.0,129.1,129.2,129.3,136.6$, $137.3,137.6,137.7,137.7,138.8,142.3,142.7,147.1,147.4$, 150.7 , 153.5, 153.6, 165.0, 167.3; IR (KBr) $v_{\max } 3064,3032$, 2947, 2874, 2360, 2342, 1704, 1684, 1591, 1497, 1426, 1375, 1217, 1092, 972, 909, 749, 736, $696 \mathrm{~cm}^{-1}$; HR-MS (FAB, positive ion mode) Calcd for $\mathrm{C}_{56} \mathrm{H}_{47} \mathrm{O}_{10}[\mathrm{M}+\mathrm{H}]^{+}:$879.3169; Found: $879.3190[\mathrm{M}+\mathrm{H}]^{+}$.

Acknowledgments This work was financially supported by the JSPS KAKENHI Grant No. 22590003 and the Tamura Science and Technology Foundation.

\section{References and Notes}

1) "Chemistry and Biology of Ellagitannins. An Underestimated Class of Bioactive Plant Polyphenols," ed. by Quideau S., World Scientific Publishing Co., Pte. Ltd., Singapore, 2009.

2) Quideau S., Feldman K. S., Chem. Rev., 96, 475-503 (1996).

3) Khanbabaee K., van Ree T., Synthesis, 1585-1610 (2001).

4) Feldman K. S., Phytochemistry, 66, 1984-2000 (2005).

5) Pouységu L., Deffieux D., Malik G., Natangelo A., Quideau S., Nat. Prod. Rep., 28, 853-874 (2011).

6) Mayer W., Liebigs Ann., 578, 33-44 (1952).

7) Hatano T., Hattori S., Okuda T., Chem. Pharm. Bull., 34, 40924097 (1986).

8) Mayer W., Fikentscher R., Chem. Ber., 91, 1542-1545 (1958)

9) Feldman K. S., Quideau S., Appel H. M., J. Org. Chem., 61, 66566665 (1996)

10) Feldman K. S., Sahasrabudhe K., J. Org. Chem., 64, 209-216 (1999).

11) Feldman K. S., Lawlor M. D., J. Am. Chem. Soc., 122, 7396-7397 (2000).

12) Feldman K. S., Lawlor M. D., Sahasrabudhe K., J. Org. Chem., 65, 8011-8019 (2000).

13) Our preliminary attempts on hydrolysis conditions to distinguish the two methyl esters were unsuccessful.

14) Ley S. V., Thomas A. W., Angew. Chem. Int. Ed., 42, 5400-5449 (2003), and references cited therein.

15) The basic examination of the Ullmann condensation was performed in our previous studies. ${ }^{16-18)}$

16) Abe H., Sahara Y., Matsuzaki Y., Takeuchi Y., Harayama T., Tetrahedron Lett., 49, 605-609 (2008).

17) Shioe K., Takeuchi Y., Harayama T., Abe H., Chem. Pharm. Bull., 
58, 435-437 (2010).

18) Shioe K., Sahara Y., Horino Y., Harayama T., Takeuchi Y., Abe H., Tetrahedron, 67, 1960-1970 (2011).

19) Pearson A. J., Bruhn P. R., J. Org. Chem., 56, $7092-7097$ (1991).

20) Ohzeki T., Mori K., Biosci. Biotechnol. Biochem., 67, 2584-2590 (2003).

21) Fuson R. C., Cleveland E. A., Org. Synth., Coll. Vol. III, 339-340 (1955).

22) Maiti D., Buchwald S. L., J. Org. Chem., 75, 1791-1794 (2010).

23) Because the homo-coupling of the aryl bromide could not be avoided, 3 eq. of 7 was necessary.

24) Tanaka M., Ikeya Y., Mitsuhashi H., Maruno M., Wakamatsu T., Tetrahedron, 51, 11703-11724 (1995).

25) Percec V., Bera T. K., Glodde M., Fu Q., Balagurusamy V. S. K.,
Heiney P. A., Chemistry, 9, 921-935 (2003).

26) Domon L., Uguen D., Tetrahedron Lett., 41, 5501-5505 (2000).

27) Alam A., Takaguchi Y., Ito H., Yoshida T., Tsuboi S., Tetrahedron, 61, 1909-1918 (2005).

28) Nawwar M. A. M., Hussein S. A. M., Buddrus J., Linscheid M., Phytochemistry, 35, 1349-1354 (1994).

29) Thapa M., Kim Y., Desper J., Chang K., Hua D. H., Bioorg. Med. Chem. Lett., 22, 353-356 (2012).

30) Ohta S., Shimabayashi A., Makino N., Okamoto M., Yakugaku Zasshi, 103, 991-993 (1983).

31) Wijesekera R. O. B., Ratnayake V. U., J. Appl. Chem. Biotechnol., 22, 559-564 (1972).

32) Xia J., Hui Y., Synth. Commun., 26, 881-886 (1996). 